\title{
Measuring Beam Sizes and Ultra-Small Electron Emittances Using an X-ray Pinhole Camera
}

\author{
P. Elleaume, C. Fortgang, ${ }^{\star}$ C. Penel and E. Tarazona \\ European Synchrotron Radiation Facility, BP 220, F-38043 Grenoble CEDEX, France
}

(Received 1 April 1995; accepted 27 June 1995)

A very simple pinhole camera set-up has been built to diagnose the electron beam emittance of the ESRF. The pinhole is placed in the air next to an Al window. An image is obtained with a CCD camera imaging a fluorescent screen. The emittance is deduced from the size of the image. The relationship between the measured beam size and the electron beam emittance depends upon the lattice functions $\alpha$, $\beta$ and $\eta$, the screen resolution, pinhole size and photon beam divergence. The set-up is capable of measuring emittances as low as $5 \mathrm{pm}$ rad and is presently routinely used as both an electron beam imaging device and an emittance diagnostic.

Keywords: beam size; electron emittance; pinhole camera; storage rings.

\section{Introduction}

A large number of third-generation synchrotron sources are presently being built or are already in operation. An important issue for these sources is high brilliance of the photon beam. For high-energy X-rays, the brilliance from an undulator is almost proportional to the inverse of the product of the horizontal and vertical electron beam emittances. Measuring the electron beam emittance is therefore one of the most important diagnostics for a synchrotron light source.

At the ESRF three different diagnostics of emittances have been developed. One of them is based on the imaging of the electron beam in a bending magnet using the visible light part of the spectrum. This diagnostic has proved to be impractical with low emittance due to the tight tolerances of mirror fabrication and its deformation under heat load from the X-ray beam. A second diagnostic involves imaging the central cone of an undulator at a photon energy of $28 \mathrm{keV}$ (Tarazona \& Elleaume, 1995). It has been successfully used for almost two years but it was found to be imprecise for emittances lower than $0.2 \mathrm{~nm}$ rad. The limitations of these diagnostics motivated the development of a third which is based on an X-ray pinhole camera. A number of attempts have already been made on measuring electron beam emittances using a pinhole camera (Jackson, Siemann \& Mills, 1983; Ogata, Mitsuhachi, Katsura, Yamamoto \& Kawamoto, 1989; Suller, 1987). In previous pinhole experiments performed on bending magnet radiation, the emittance of the beam was rather large, allowing the full measurement of the vertical emittance through both the size and angular spread measurement. With the small emittance

\footnotetext{
* Present address: Los Alamos National Laboratory, New Mexico, USA.
}

beam of the ESRF, the divergence of the bending magnet radiation is fully dominated by the divergence of the radiation generated by a single electron through the process of synchrotron radiation. In other words, the vertical emittance of the photon beam from a bending magnet is much larger than the emittance of the electron beam. Nevertheless, knowing the Twiss parameters of the lattice at the source point it is possible to deduce the electron beam emittance from a measured beam size. This paper describes our set-up, the theoretical framework required to interpret the measurement and the results obtained.

\section{Description}

The X-ray pinhole camera has the same geometrical properties as the more familiar visible-light pinhole camera. From each source point, one ray passes through the pinhole (if it is infinitesimally small) forming an inverted image of the source. A layout of the apparatus is shown in Fig. 1. The $\mathrm{X}$-ray source is the synchrotron radiation coming from the electron beam at the entrance of a bending magnet. The $\mathrm{X}$-rays exit the storage ring vacuum through a $3 \mathrm{~mm}$ thick $\mathrm{Al}$ window. The pinhole is $1 \mathrm{~m}$ downstream of the window and $4.45 \mathrm{~m}$ downstream of the source point. After passing through the pinhole, the $\mathrm{X}$-rays strike a fluorescent screen located $11.57 \mathrm{~m}$ downstream of the pinhole. Finally, the visible light is imaged with a CCD camera. The pinhole does not require cooling. The magnetic field at the source point has been chosen to be $0.4 \mathrm{~T}$ in the fringe field of the bending magnet, which has a $0.85 \mathrm{~T}$ nominal field. This presents two advantages. The heat load on the $\mathrm{Al}$ window is reduced and the dispersion function of the lattice (which complicates the interpretation of the measurement) is not as 
large as in the dipole. Most of the X-rays from the bending magnet are absorbed by a water-cooled $\mathrm{Cu}$ block that resides in the vacuum of the ring. The heat load is reduced further by the water-cooled $\mathrm{Al}$ window, leaving only about $20 \mathrm{~W}$ to be absorbed by the pinhole which does not require any water cooling. The remotely movable $\mathrm{Cu}$ attenuator, just in front of the pinhole, is used to prevent signal saturation of the CCD camera and not for protection of the pinhole assembly. The X-ray intensity from the source can vary by a factor of 30 depending on the electron current stored in the ring.

The pinhole is quite simple and easy to fabricate. There are actually $3 \times 3$ rectangular pinholes of various sizes. They are formed using a grid of flat and parallel carbide tungsten plates separated by Al spacers (Fig. 2). The plates are machined to a flatness of $2 \mu \mathrm{m}$. The pinhole assembly is remotely moveable with four degrees of freedom: horizontal $(x)$ translation, vertical $(z)$ translation, rotation around the $x$ axis, and rotation around the $z$ axis. For the pinhole, one can choose among three horizontal $(400,100$ or $50 \mu \mathrm{m})$ and three vertical $(400,50$ and $25 \mu \mathrm{m})$ sizes. The $400 \times 400 \mu \mathrm{m}$ pinhole is used for alignment purposes. Note that the nonzero penetration of hard $\mathrm{X}$-rays (critical photon energy of $9.6 \mathrm{keV}$ ) in tungsten requires the use of a thick pinhole. We are using a $5.5 \mathrm{~mm}$ thick carbide tungsten even though $2 \mathrm{~mm}$ was expected to be sufficient. Because of the thickness of the pinhole one should view it like a tunnel rather than a simple hole. With the smallest size aperture, the tunnel presents an aspect ratio of 220 which requires a precise angular adjustment to place it parallel to the incoming $\mathrm{X}$-ray beam. Note that a non-parallelism of the beam with the tunnel axis results in an effective reduction of the aperture. This feature, together with an over-dimensioned thickness (factor of 3), allows for further reduction of the pinhole size by rotating it around the horizontal and/or vertical axis. The optical light from the fluorescent screen is reflected $90^{\circ}$ with a mirror and imaged by a CCD camera. The mirror provides a means of keeping the camera out of the direct line-of-sight of the X-ray beam. For alignment purposes, the screen, mirror and CCD camera are mounted together on an $x-z$ translation stage. The $x-z$ translation, camera focus and camera integration time are remotely controlled. Two fluorescent screens have been tried, Kodak Min-R $\left(\mathrm{Gd}_{2} \mathrm{O}_{2} \mathrm{~S}: \mathrm{Tb}\right)$ and Harshaw Bicron cadmium tungstate $\left(\mathrm{CdWO}_{4}\right)$. The cadmium tungstate is used because it is more radiation resistant even though it gives about three. times less signal. Pinhole, attenuator and camera are placed in the ring tunnel. The pinhole and camera assemblies are initially aligned using an $\mathrm{HeNe}$ laser. The laser is prealigned to the orbit plane of the machine and the straight section directly upstream of the bending magnet. The assemblies are then horizontally moved towards the inside of the ring to image the synchrotron radiation with the $400 \times 400 \mu \mathrm{m}$ pinhole. The final alignment is made with the $\mathrm{X}$-ray beam. Smaller pinholes are successively moved into position and small adjustments of position and angle are made to maximize the image intensity. The image is displayed on a colour monitor and size measurements are made in real time using image processing. After digitization, the image is fitted to a two-dimensional Gaussian profile from which, among other coefficients, the horizontal and vertical r.m.s. sizes of the photon beam are extracted (Tarazona \& Elleaume, 1995).

\section{Theory}

In this section we present the model which we use to calculate the electron-beam emittance from the r.m.s. size of the visible-light image obtained with the CCD camera. We assume uncoupled horizontal and vertical betatron oscillations which allows us to treat the size and divergence in the horizontal and vertical planes independently. In each plane, the size and divergence of the stored electron beam are defined using six independent quantities: the emittance $\varepsilon$, the values of the lattice parameters $\beta$ and $\alpha$ (Courant $\&$ Snyder, 1958), the dispersion $\eta$, the derivative of the dispersion function $\eta^{\prime}$, and the relative energy spread $\sigma_{e}$. Note that of these six quantities only the energy spread is common to both the horizontal and the vertical planes. The parameters $\beta, \alpha, \eta$ and $\eta^{\prime}$ depend on the longitudinal position $s$ along the circumference of the ring.

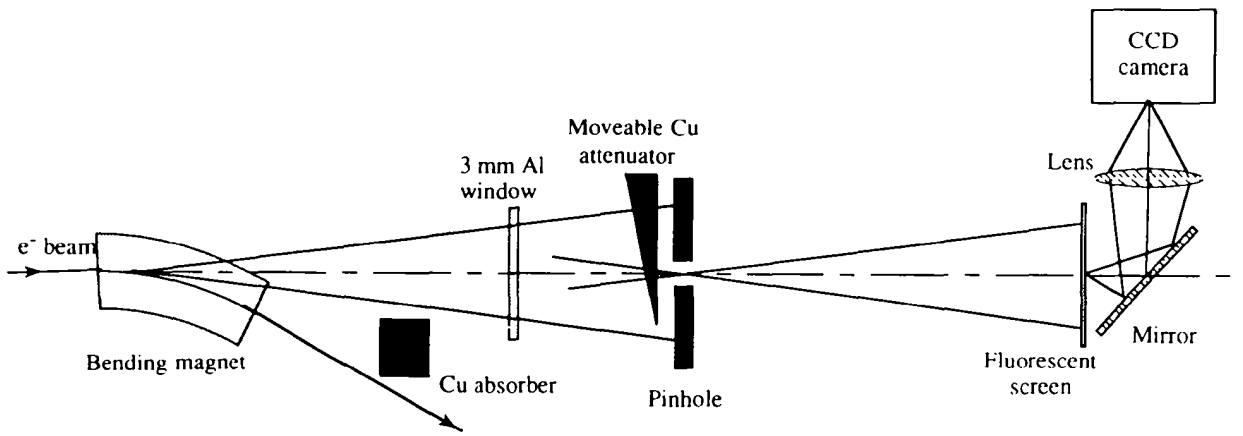

Figure 1

Schematic of the pinhole camera apparatus and the X-ray source. 
Let $y_{e}$ and $y_{e}^{\prime}$ be the position and angle of an electron (in one plane) at a position $s$. We define $\langle f\rangle$ as the statistical average over all the electrons in the beam of the physical quantity $f$. The quantities $\left\langle y_{e}^{2}\right\rangle,\left\langle y_{e}^{\prime 2}\right\rangle$ and $\left\langle y_{e} y_{e}^{\prime}\right\rangle$ are expressed in terms of the previously defined parameters (Sands, 1971) as:

$$
\begin{aligned}
\left\langle y_{e}^{2}\right\rangle & =\beta \varepsilon+\left(\eta \sigma_{e}\right)^{2} \\
\left\langle y_{e}^{\prime 2}\right\rangle & =\gamma \varepsilon+\left(\eta^{\prime} \sigma_{e}\right)^{2} \\
\left\langle y_{e} y_{e}^{\prime}\right\rangle & =-\alpha \varepsilon+\eta \eta^{\prime} \sigma_{e}^{2}
\end{aligned}
$$

where $\gamma$ is defined as,

$$
\gamma=\left(1+\alpha^{2}\right) / \beta
$$

The synchrotron radiation process creates a photon beam associated with each individual electron. Let us define the quantities $y_{p}$ and $y_{p}^{\prime}$ as the position and angle of photon radiated by a single electron. The independence of the radiation process from one electron to the next allows us to write:

$$
\begin{aligned}
\left\langle y^{2}\right\rangle & =\left\langle y_{e}^{2}\right\rangle+\left\langle y_{p}^{2}\right\rangle \\
\left\langle y^{\prime 2}\right\rangle & =\left\langle y_{e}^{\prime 2}\right\rangle+\left\langle y_{p}^{\prime 2}\right\rangle \\
\left\langle y y^{\prime}\right\rangle & =\left\langle y_{e} y_{e}^{\prime}\right\rangle+\left\langle y_{p} y_{p}^{\prime}\right\rangle
\end{aligned}
$$

where $y$ and $y^{\prime}$ refer to the position and angle of any photon emitted by the whole electron beam. Two terms in these equations are negligible. First, the symmetry of the emission process imposes $\left\langle y_{p} y_{p}^{\prime}\right\rangle=0$. Second, we neglect $\left\langle y_{p}^{2}\right\rangle$ for the following reason. For a photon beam of wavelength $\lambda$, diffraction imposes, at the source point, the following condition:

$$
\left\langle y_{p}^{2}\right\rangle\left\langle y_{p}^{\prime 2}\right\rangle \geq(\lambda / 4 \pi)^{2} .
$$

We use this equation to estimate $\left\langle y_{p}^{\prime 2}\right\rangle\left[\left(\left\langle y_{p}^{\prime 2}\right\rangle\right)^{1 / 2}\right.$ is of the order of $1 / \gamma \simeq 1 \times 10^{-4}$ for ESRF and $\lambda$ is of the order of $\left.10^{-10} \mathrm{~m}\right]$ and find it to be negligible compared to $\left\langle y_{e}^{2}\right\rangle$.

The photon beam emittance $E$ is defined as:

$$
E^{2}=\left\langle y^{2}\right\rangle\left\langle y^{\prime 2}\right\rangle-\left\langle y y^{\prime}\right\rangle^{2} .
$$

The generalized Twiss parameters $A, B$ and $G=\left(1+A^{2}\right) / B$

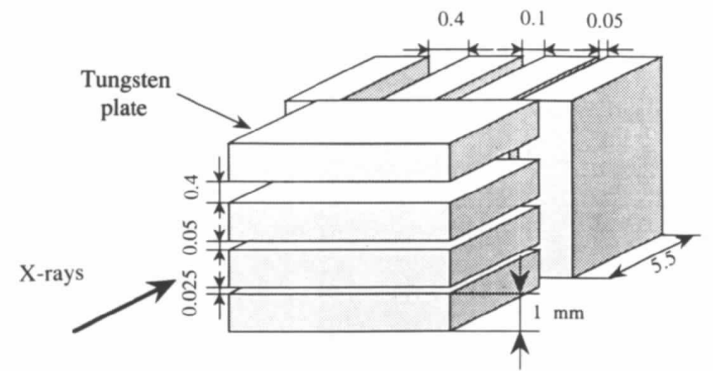

Figure 2

An array of $3 \times 3$ rectangular pinholes is made with tungsten plates. The wide pinhole is used for alignment purposes using an HeNe laser. The small pinholes are used during the measurement. of the photon beam are defined as:

$$
\begin{aligned}
& B E=\left\langle y^{2}\right\rangle \cong \beta \varepsilon+\left(\eta \sigma_{e}\right)^{2} \\
& G E=\left\langle y^{\prime 2}\right\rangle \cong \gamma \varepsilon+\left(\eta^{\prime} \sigma_{e}\right)^{2}+\left\langle y_{p}^{\prime 2}\right\rangle \\
& A E=-\left\langle y y^{\prime}\right\rangle \cong \alpha \varepsilon-\eta \eta^{\prime} \sigma_{e}^{2} .
\end{aligned}
$$

We now calculate the size of the image assuming a Gaussian beam. The distribution function $f\left(y, y^{\prime}\right)$ of the photon beam at the source point is given by:

$$
f_{\text {source }}\left(y, y^{\prime}\right)=(1 / 2 \pi E) \exp \left[-\left(G y^{2}+2 A y y^{\prime}+B y^{\prime 2}\right) / 2 E\right] .
$$

After transforming $y$ and $y^{\prime}$ to the pinhole, located a distance $d$ from the source, we obtain the distribution function $f\left(y, y^{\prime}\right)$ of the photon beam at the pinhole:

$$
\begin{aligned}
f_{\text {pinhole }}\left(y, y^{\prime}\right)= & (1 / 2 \pi E) \exp \left\{-\left[G y^{2}+2(A+d G) y y^{\prime}\right.\right. \\
& \left.\left.+\left(B+2 A d+G d^{2}\right) y^{\prime 2}\right] / 2 E\right\} .
\end{aligned}
$$

Let $S$ be the r.m.s. size of the photon beam at the screen located at a distance $D$ from the pinhole. In defining $S$ we neglect for the moment the effects of finite pinhole size, diffraction at the pinhole, and broadening of the image due to screen and camera resolution. $S$ is equal to the r.m.s. divergence of the beam at the centre of the pinhole multiplied by $D$. The r.m.s. divergence of the photon beam at the centre of the pinhole is obtained from (8) after setting $y$ equal to zero. We therefore write $S$ as:

$$
S=D\left[E /\left(B+2 A d+G d^{2}\right)\right]^{1 / 2} .
$$

After combining (5), (6) and (9) we derive the electron emittance:

$$
\varepsilon=\left\{c-a+\left[(c-a)^{2}-4(b-e)\right]^{1 / 2}\right\} / 2
$$

where $a, b, c$ and $e$ are given by:

$$
\begin{aligned}
a & =\sigma_{e}^{2}\left(\eta^{2} \gamma+\eta^{\prime 2} \beta+2 \eta \eta^{\prime} \alpha\right)+\beta\left\langle y_{p}^{\prime 2}\right\rangle \\
b & =\sigma_{e}^{2} \eta^{2}\left\langle y_{p}^{\prime 2}\right\rangle \\
c & =\left(S^{2} / D^{2}\right)\left(\beta+2 \alpha d+\gamma d^{2}\right) \\
e & =\left(S^{2} / D^{2}\right)\left[\left(\eta-\eta^{\prime} d\right)^{2} \sigma_{e}^{2}+d^{2}\left\langle y_{p}^{\prime 2}\right\rangle\right] .
\end{aligned}
$$

In (10), $c$ is directly related to the emittance while $a, b$ and $e$ are unfavourable terms introduced by the photon divergence and dispersion. Their contribution to the measurement will be discussed in the next section.

Finally, we define $\Sigma$ as the r.m.s. size of the image obtained after the image processing. It is the sum of several independent r.m.s. contributions,

$$
\Sigma=\left(S^{2}+S_{\text {screen }}^{2}+S_{\text {camera }}^{2}+S_{\text {diffraction }}^{2}+S_{\text {pinhole }}^{2}\right)^{1 / 2} .
$$

$S_{\text {screen }}$ is the screen spatial resolution. $S_{\text {camera }}$ is the spread induced by the camera; it includes pixel size, lens aberration and depth of focus through the finite thickness of the screen and aperture of the lens. $S_{\text {diffraction }}$ is the diffraction contribution by the small pinhole. $S_{\text {pinhole }}$ is the geometrical contribution introduced by the finite size of the pinhole. 
Geometrical and diffraction effects from the pinhole are estimated as follows. Let $w$ be the aperture of a rectangular shaped pinhole. A simple geometrical computation shows that $S_{\text {pinhole }}$ can be expressed as:

$$
S_{\text {pinhole }}=[w(D+d)] /(12)^{1 / 2} d .
$$

Applying (4) gives an estimate for $S_{\text {diffraction }}$ for a monochromatic photon beam of wavelength $\lambda$ :

$$
S_{\text {diffraction }} \simeq\left[(12)^{1 / 2} / 4 \pi\right](\lambda D / w) .
$$

Equations (10)-(14) relate the electron beam emittance to the r.m.s. size measured on the image of the camera. One needs to know a large number of parameters, in addition to measuring the r.m.s. size of the image, to deduce the electron beam emittance. Imprecisions in the measurement of these parameters are responsible for the final precision on the computed emittance.

Our treatment of the problem has been quite general. We now look at a special case. Consider $\left\langle y_{p}^{\prime 2}\right\rangle=\infty$, then $S$ is given by:

$$
S=(D / d)\left[\beta \varepsilon+\left(\eta \sigma_{e}\right)^{2}\right]^{1 / 2}=(D / d) \sigma
$$

where $\sigma$ is the size of the source. This is the usual pinhole camera result where the image is equal to the size of the source enlarged by the ratio of the distances screen-topinhole and source-to-pinhole. A further approximation is $\eta=0$ in which case the dependence between $S$ and $\varepsilon$ only requires the measurement of $d$ and $D$, which are straightforward, and $\beta$, which is more delicate. In practice $\left\langle y_{p}^{\prime 2}\right\rangle$ may not be infinite and one may observe significant deviations from (15). One can understand the importance of the source divergence from the following argument. Consider a photon beam with no divergence at all. After passing through an infinitely small pinhole the beam maintains zero divergence (neglecting diffraction) and therefore projects an infinitely small size at the screen, violating (15). A detailed analysis shows that (15) gives an upper limit to the measured size.

In a bending magnet, the curvature of the electron trajectory in the horizontal plane, under the action of the vertical magnetic field, results in an effective infinite horizontal divergence at the source. Therefore, (15) applies in the horizontal plane. Large divergence is not the case in the vertical plane and we have found practical cases were (15) is strongly violated. In the next section we shall apply the above analysis to our specific pinhole set-up.

\section{Results}

Fig. 3 shows an image taken with the CCD camera. The fit to a two-dimensional Gaussian is in all cases excellent. With a $50 \times 25 \mu \mathrm{m}$ pinhole, one can record a good-quality image for any stored electron current higher than $4 \mathrm{~mA}$. Lower current can be studied with larger pinholes. For pinholes that are not too large, the signal is roughly proportional to the pinhole surface. The dynamic range is obtained by changing
Table 1

Values for the parameters used in the emittance calculation, the measured beam size and the calculated emittance.

\begin{tabular}{lcl}
\hline & Horizontal & Vertical \\
\hline$\beta(\mathrm{m})$ & 2.83 & 26.2 \\
$\alpha$ & 1.63 & 0.06 \\
Dispersion $(\mathrm{m})$ & 0.031 & 0 \\
Dispersion derivative $(\mathrm{m})$ & -0.024 & 0 \\
Energy spread & 0.001 & 0.001 \\
$\left(\left\langle y_{p}^{\prime 2}\right\rangle\right)^{1 / 2}(\mu \mathrm{r})$ & $\infty$ & 18.5 \\
Pinhole size $(\mu \mathrm{m})$ & 50 & 25 \\
$S_{\text {pinhole }}(\mu \mathrm{m})$ & 52 & 26 \\
$S_{\text {diffraction }}(\mu \mathrm{m})$ & $\sim 2$ & $\sim 4$ \\
$S_{\text {screen }}(\mu \mathrm{m})$ & $\sim 30$ & $\sim 30$ \\
Measured r.m.s. size $\Sigma(\mu \mathrm{m})$ & 277 & 89 \\
Emittance from pinhole $(\mathrm{nm} \mathrm{rad})$ & 3.5 & 0.041 \\
Emittance from ID6 $(\mathrm{nm} \mathrm{rad})$ & 4.0 & 0.06 \\
\hline
\end{tabular}

the integration time of the CCD camera between 0.1 and $20 \mathrm{~ms}$ and by moving the copper attenuator in and out. Table 1 presents the important parameters and the measured r.m.s. beam sizes in March 1995. The lattice functions $\beta, \alpha, \eta, \eta^{\prime}$ and energy spread are the design calculated values. To insure that the lattice functions of the source point are as close as possible to the design value, the horizontal and vertical half-integer resonances $\left(2 v_{x}=73\right.$ and $\left.2 v_{z}=23\right)$ in the vicinity of the operating tune $\left(v_{x}=36.44, v_{z}=11.37\right)$ were corrected using localized quadrupolar correction. The pinhole sizes were chosen after an optimization between flux (large aperture) and resolution (small aperture). The contribution from diffraction assumes a typical photon energy of $40 \mathrm{keV}$. The divergence of the single electron emission $\left(\left\langle y_{p}^{\prime 2}\right\rangle\right)^{1 / 2}$. was measured by removing the pinhole and measuring the vertical size of the spot on the camera. Note that the natural divergence of the photon beam should scale like $1 / \gamma$ which is $85 \mu \mathrm{rad}$ for our electron energy of $6 \mathrm{GeV}$. The measured value is more than four times smaller due to the hardening of the radiation by the $\mathrm{Al}$ window, the $\mathrm{Cu}$ attenuator and the air absorption. The contribution from the screen resolution was measured by moving the pinhole and the camera horizontally in such a way to observe another source point: the upstream dipole

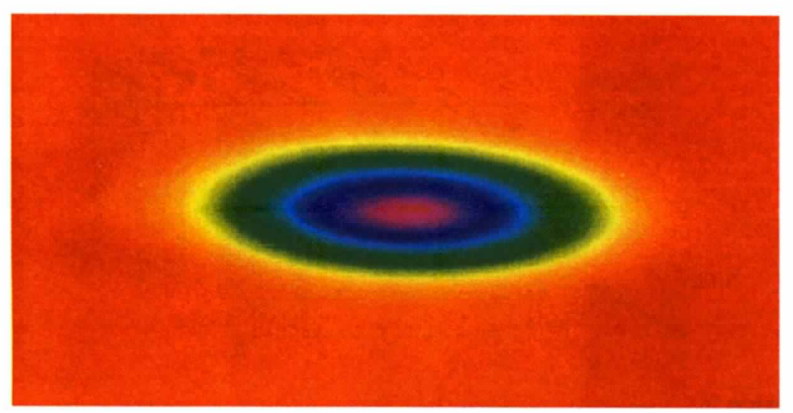

Figure 3

Typical image of the electron beam obtained with the pinhole camera. 
which is located at about $23 \mathrm{~m}$ from the pinhole. Owing to the demagnification which occurs with the large sourcepinhole separation, the image from this second source point is much smaller. The minimum size measured after optimizing the focusing of the CCD camera is taken as the screen resolution but it also includes the aberration of the lens. Finally, the emittance is computed using equations (10) and (11).

We have compared the computed pinhole camera emittance with those measured by another emittance diagnostic. This other diagnostic is based on the observation of the central cone of the machine diagnostics undulator ID6 (Tarazona \& Elleaume, 1995). There is a good agreement in the horizontal plane. Some discrepancy is observed in the vertical plane. This is expected since the ID6 diagnostic is not precise for low-emittance beams $(<0.2 \mathrm{~nm} \mathrm{rad})$. Note that these measurements are in good agreement with the design horizontal emittance of $4 \mathrm{nmrad}$. The smallest achievable vertical emittance depends on the residual positioning errors of the quadrupoles and sextupoles in the ring together with the level of correction of the lattice. The emittance measurement was performed after a minimization of the closed orbit error below $0.1 \mathrm{~mm}$ r.m.s. in both horizontal and vertical planes and correction of the two coupling resonances $\left(v_{x}+v_{z}=48\right.$ and $\left.v_{x}-v_{z}=25\right)$ in the direct vicinity of the operating tune by means of skew quadrupoles. The smallest vertical size was found almost to coincide with the minimum width of these resonances. Fig. 4 presents the relation between the measured r.m.s. beam size and the calculated emittance using the parameters of Table 1. The slopes of these curves are related to the precision of the measurement. When the slope is low, any imprecision in the measurement of the size or imprecision in the knowledge of the pinhole size and screen resolution results in a large uncertainty in the value of the deduced emittance. Fortunately, the present point of operation, indicated on the figure, corresponds to a significant slope resulting in a maximum precision in the computation of the

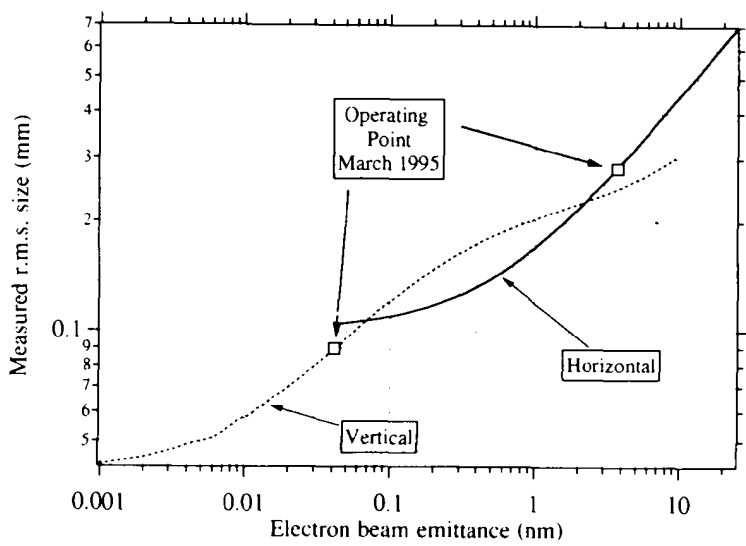

Figure 4

Results of the model which calculates the electron beam emittance from the measured size of the electron beam.
Table 2

Uncertainty in the parameters for the emittance calculation and the uncertainty in the final result.

\begin{tabular}{lcc}
\hline & $\begin{array}{c}\text { Horizontal } \\
\text { emittance }(\%)\end{array}$ & $\begin{array}{c}\text { Vertical } \\
\text { emittance }(\%)\end{array}$ \\
\hline $10 \%$ precision on $\beta$ & 9 & 9 \\
$10 \%$ precision on $\left(\left\langle y_{p}^{\prime}{ }^{2}\right\rangle\right)^{1 / 2}$ & 0 & 3 \\
$10 \%$ on the screen resolution & 0 & 4 \\
$30 \%$ on the energy spread & 7 & 0 \\
$5 \%$ on the measured size & 12 & 15 \\
Total & 17 & 18 \\
\hline
\end{tabular}

emittance. The non-zero energy spread of the electron beam is responsible for a small broadening of the measured horizontal beam size at the camera location. The absence of energy spread would have lead to a horizontal beam size on the CCD of $0.265 \mathrm{~mm}$ (instead of the measured $0.277 \mathrm{~mm}$ ). Similarly, an infinite photon beam divergence $\left\langle y_{p}^{\prime 2}\right\rangle$ would have lead to a vertical beam size on the CCD of $0.094 \mathrm{~mm}$ (instead of the measured $0.089 \mathrm{~mm}$ ). In other words, the terms $a, b$ and $e$ in (10) make a small perturbation. For the present operating point, Table 2 summarizes the main sources of error on the computed emittances. They originate from the imprecise knowledge of the beta functions (10\%), the screen resnlution (10\%), the single electron divergence $(10 \%)$, the energy spread $(30 \%)$ and the size measurement itself $(5 \%)$. This table has been computed by means of equations (10)-(13). Therefore, we conclude that the measured emittance has an accuracy better than $20 \%$. If the vertical emittance is decreased, one could still measure it, but with a lower precision. A precision of $50 \%$ should be possible using the same pinhole, screen and camera for an emittance of $5 \mathrm{pm}$ rad. We estimate that the error could be reduced to $25 \%$ by using a smaller pinhole and by using a screen with higher resolution. The optimal pinhole size minimizes the effects of diffraction and geometrical broadening. For our set-up, this size is $c a 10 \mu \mathrm{m}$. We therefore conclude that our emittance diagnostic is capable of detecting emittances more than ten times smaller than that presently observed.

\section{Conclusions}

A pinhole camera diagnostic has been built at the ESRF. It is almost aberration free and gives a high-quality image of the electron beam at the entrance of a dipole. Horizontal and vertical emittances of 3.5 and $0.04 \mathrm{~nm} \mathrm{rad}$ have been measured. It is capable of diagnosing emittances as low as $5 \mathrm{pm}$ rad even though, to our knowledge, such emittances have not yet been observed on any storage ring anywhere in the world. It is presently routinely used as both a beam imaging device at the video frequency of the CCD camera and as an emittance diagnostic. Our implementation of the pinhole camera makes full use of the transparency of the air and aluminium to the hard $\mathrm{X}$-rays, resulting in an extremely 
simple and inexpensive set-up which outperforms all our other electron beam imaging devices.

\section{References}

Courant, E. D. \& Snyder, H. S. (1958). Ann. Phys. 3, 1-18.

Jackson, G. P., Siemann, R. H. \& Mills, D. N. (1983). Proceedings of the 12th Conference on Higher Energy Accelerators, p. 217.
Ogata, A., Mitsuhachi, T., Katsura, T., Yamamoto, N. \& Kawamoto, T. (1989). Proceedings of the 1989 IEEE Particle Accelerator Conference, p. 1498. New York: IEEE.

Sands, M. (1971). The Physics of Electron Storage Rings. Proceedings of the International School 'Enrico Fermi', Vol. 46, p. 258.

Suller, V. P. (1987). Private communication.

Tarazona, E. \& Elleaume, P. (1995). Rev. Sci. Instrum. 66(2), 1974. 